\title{
Control of Chiral Magnetism Through Electric Fields in Multiferroic Compounds above the Long-Range Multiferroic Transition
}

\author{
J. Stein, ${ }^{1}$ M. Baum, ${ }^{1, \dagger}$ S. Holbein, ${ }^{1,2}$ T. Finger, ${ }^{1}$ T. Cronert, ${ }^{1}$ C. Tölzer, ${ }^{1}$ T. Fröhlich, ${ }^{1}$ \\ S. Biesenkamp, ${ }^{1}$ K. Schmalzl, ${ }^{3}$ P. Steffens, ${ }^{2}$ C. H. Lee, ${ }^{4}$ and M. Braden ${ }^{1, *}$ \\ ${ }^{1}$ II. Physikalisches Institut, Universität zu Köln, Zülpicher Straße 77, 50937 Köln, Germany \\ ${ }^{2}$ Institut Laue-Langevin, 71 avenue des Martyrs, 38000 Grenoble, France \\ ${ }^{3}$ Jülich Centre for Neutron Science, Forschungszentrum Jülich GmbH, \\ Outstation at Institut Laue-Langevin, 71 avenue des Martyrs, 38000 Grenoble, France \\ ${ }^{4}$ National Institute of Advanced Industrial Science and Technology (AIST), Tsukuba, Ibaraki 305-8568, Japan
}

(Received 24 March 2017; published 26 October 2017)

\begin{abstract}
Polarized neutron scattering experiments reveal that type-II multiferroics allow for controlling the spin chirality by external electric fields even in the absence of long-range multiferroic order. In the two prototype compounds $\mathrm{TbMnO}_{3}$ and $\mathrm{MnWO}_{4}$, chiral magnetism associated with soft overdamped electromagnons can be observed above the long-range multiferroic transition temperature $T_{\mathrm{MF}}$, and it is possible to control it through an electric field. While $\mathrm{MnWO}_{4}$ exhibits chiral correlations only in a tiny temperature interval above $T_{\mathrm{MF}}$, in $\mathrm{TbMnO}_{3}$ chiral magnetism can be observed over several kelvin up to the lock-in transition, which is well separated from $T_{\mathrm{MF}}$.
\end{abstract}

DOI: 10.1103/PhysRevLett.119.177201

Multiferroic materials with coupled magnetic and ferroelectric order bear considerable application potential [1,2]. In type-II multiferroics, magnetic order directly induces ferroelectric polarization and giant magnetoelectric coupling. External magnetic fields imply a flop of electric polarization, and electric fields can control chiral magnetic domains [1-5]. Various neutron experiments [6-12] as well as resonant and nonresonant $\mathrm{X}$-ray studies $[13,14]$ show that cooling in electric fields enforces a monodomain chiral state, and varying external electric fields at constant temperature drives the chiral magnetic order [9-12], which corresponds to the most promising application as a data storage medium. In addition, time resolved soft $\mathrm{x}$-ray diffraction showed that chiral magnetism can be manipulated by THz-radiation pulses at an electromagnon energy [15].

So far, studies of the multiferroic coupling and hysteresis curves were restricted to the phases with long-range magnetic order on bulk or film materials [16], while only small multiferroic blocks would be vital for applications. Also, from the fundamental point of view, one may ask whether multiferroic hysteresis and control can be achieved in short-range systems above the long-range static multiferroic transition, and how far spin chirality persists above the static and long-range multiferroic order. The mixed system $\mathrm{Ni}_{0.42} \mathrm{Mn}_{0.58} \mathrm{TiO}_{3}$ already indicates that magnetoelectric coupling can persist in cluster systems with competing magnetic structures [17], but until now there has been no information about the control and multiferroic coupling of chiral ordering that is limited in space and time. Here, we study two prototype type-II multiferroics, $\mathrm{TbMnO}_{3}[1,3,4]$ and $\mathrm{MnWO}_{4}$ [18-20], above the long-range ferroelectric transition at zero electric field $T_{\mathrm{MF}}$, where it is still possible to pole and control chiral magnetic correlations. Although the two materials exhibit a similar sequence of magnetic transitions, it turns out that only in $\mathrm{TbMnO}_{3}$ can chiral scattering be controlled over a large temperature interval of several kelvin.

$\mathrm{TbMnO}_{3}\left(\mathrm{MnWO}_{4}\right)$ both exhibit a first magnetic transition at $T_{N}=42 \mathrm{~K}(13.5 \mathrm{~K})$, followed by a second transition at lower temperature, at which cycloid order develops at $T_{\mathrm{MF}}=27.6 \mathrm{~K}(12.6 \mathrm{~K})$. For both materials, polarized neutron and $\mathrm{x}$-ray scattering demonstrated the possibility to pole the long-range chiral domains upon cooling $[6,7,13]$ and to drive full multiferroic hysteresis cycles at constant temperature $[9,12]$ revealing rather slow domain inversion times in the case of $\mathrm{MnWO}_{4}$ [21]. In addition, poling and control of multiferroic domains was studied for $\mathrm{TbMnO}_{3}$ also on thin films [16].

The experimental details of our neutron diffraction experiments are given in the Supplemental Material [22]. In general, neutron scattering only senses the magnetization components perpendicular to the scattering vector. The neutron polarization analysis adds another selection rule because magnetization components perpendicular (parallel) to the neutron polarization yield a spin-flip (non-spin-flip) process [25]. Therefore, the neutron polarization allows one to separate the different magnetic components. We use the conventional coordinate system with $\boldsymbol{x}$ parallel to the scattering vector, $z$ vertical to the scattering plane, and $\boldsymbol{y}=\boldsymbol{z} \times \boldsymbol{x}$, as it is illustrated in the inset of Fig. 1. The simplest way to detect the chiral component to magnetic scattering is to compare the spin-flip scattering in the $\sigma_{x \bar{x}}$ and $\sigma_{\bar{x} x}$ channels (the indices indicate the direction of 


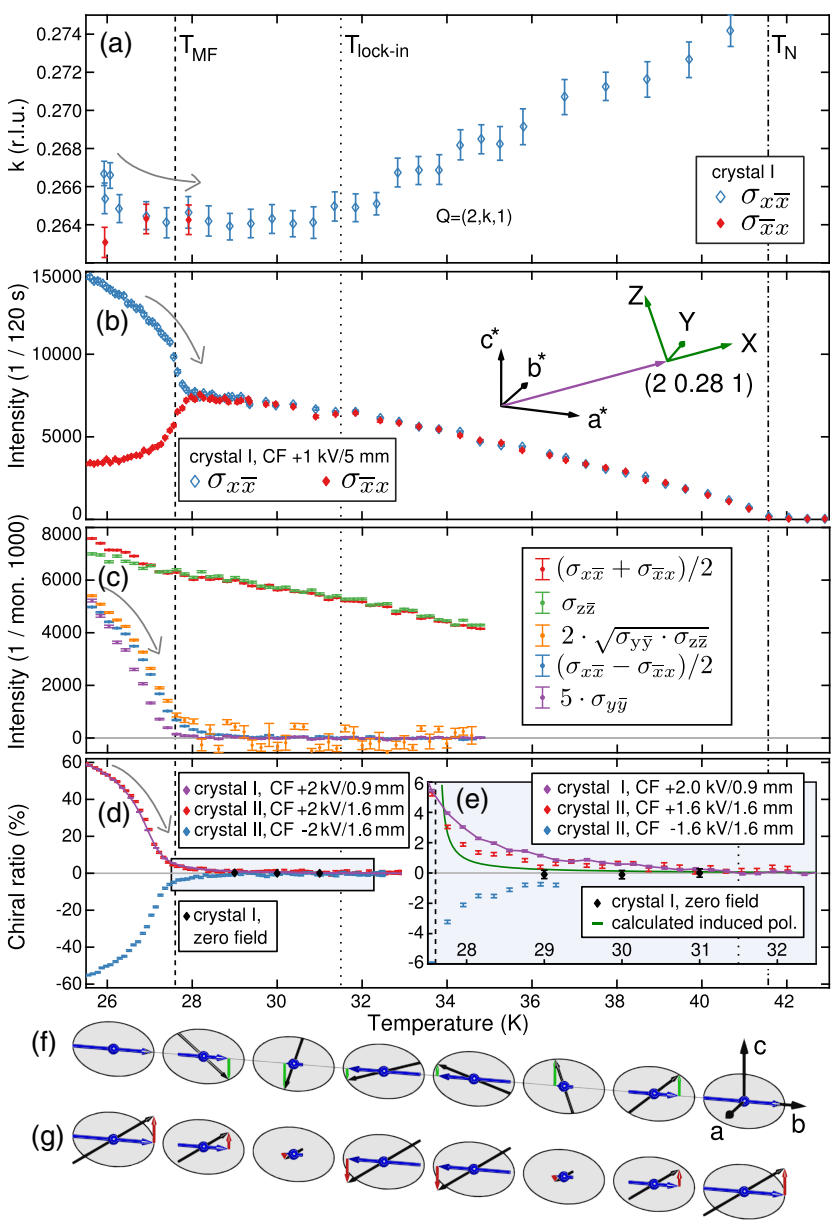

FIG. 1. Temperature dependence of the incommensurate modulation vector in $\mathrm{TbMnO}_{3}$ (a) compared to the onset of long-range chiral magnetism at $T_{\mathrm{MF}}=27.6 \mathrm{~K}$. The chiral magnetic order is determined by the difference of the polarized neutron intensities in the $\sigma_{x \bar{x}}$ and $\sigma_{\bar{x} x}$ channels shown in (b). The inset shows the coordinate system of the neutron polarization. Panel (c) compares various intensities, the chiral component $\left(\sigma_{\bar{x} x}-\sigma_{x \bar{x}}\right) / 2$ (blue), the total magnetic scattering $\left(\sigma_{\bar{x} x}+\sigma_{x \bar{x}}\right) / 2$ (red), the $c$ component $\cong \sigma_{y \bar{y}}$ corrected for the finite flipping ratio and enhanced by a factor 5 (magenta), the $b$ component $\cong \sigma_{z \bar{z}}$ (green) and $2 \sqrt{\sigma_{z \bar{z}} \sigma_{y \bar{y}}}$ (orange). (d) and (e) present the chiral ratio at the peak position for different poling fields. For high enough field, chiral magnetism persists well above $T_{\mathrm{MF}}$ and can be followed at least till $T_{\text {lock-in }}$. The green line in (e) denotes an upper limit of the longrange static chiral component that can be induced through the multiferroic coupling; see the Supplemental Material [22]. A few zero-field data points are included in (c) and (d). Insets (f) and (g) illustrate the two $c$-polarized magnons in the SDW phase (blue arrows: static moments); only the out-of-phase mode (f) with respect to the $b$ order results in a cycloid generating ferroelectric polarization.

neutron polarization, and the overlines indicate reversed polarization) [9]. Since the chiral component enters the scattering from $x$ to $\bar{x}$ and the opposed one (from $\bar{x}$ to $x$ ) with opposite signs, we may define the chiral ratio by $r_{\text {chiral }}=\left(\sigma_{x \bar{x}}-\sigma_{\bar{x} x} / \sigma_{x \bar{x}}+\sigma_{\bar{x} x}\right)$, which can be corrected for the finite neutron polarization [22]. In most cases, the chiral ratio will differ from \pm 1 even in a mono-domain ideal cycloid because the chiral component does not constitute the full magnetic scattering. For instance, it is important to measure at a $\mathbf{Q}$ vector almost perpendicular to the cycloid plane to obtain a large chiral ratio. For $\mathrm{TbMnO}_{3}\left[\mathrm{MnWO}_{4}\right]$, we studied the $\mathbf{Q}=(2,0.28,1) \quad[\mathbf{Q}=(-0.214,0.5$, 0.457)] Bragg peaks in the scattering plane determined by $(2,0,1) /(0,1,0)[(-0.214,0,0.457) /(0,1,0)]$. Since the magnetic Bragg peaks are separated from the nuclear ones, the scattering at these $\mathbf{Q}$ positions is entirely magnetic, and for determining the chiral components, it is not necessary to analyze the neutron polarization after the sample, which yields considerably higher intensity.

Figure 1 shows the temperature dependence of the incommensurate modulation compared with that of the chiral intensity upon poling $\mathrm{TbMnO}_{3}$ in electric fields. With this simultaneous measurement of both properties, one immediately recognizes the considerable offset of the locking-in of the modulation $T_{\text {lock-in }}=31.6 \mathrm{~K}$ and the onset of long-range chiral and ferroelectric order at $T_{\mathrm{MF}}=27.6 \mathrm{~K}$. Evidence for an additional thermodynamic feature above $T_{\mathrm{MF}}$ has been deduced from thermal expansion measurements in good agreement with our result [26], but, surprisingly, a clear analysis of both transitions by neutron diffraction techniques was never performed, although previous reports agree with our conclusion $[27,28]$. The lock-in phenomenon has sometimes been associated with the multiferroic transition $[3,4,29]$, but it occurs significantly, $\Delta T=4 \mathrm{~K}$, above the onset of longrange chiral and ferroelectric order.

Figures 1(b)-1(e) analyze the chiral scattering in $\mathrm{TbMnO}_{3}$ upon poling samples cut from two distinct crystal growths. In $\mathrm{TbMnO}_{3}$, it is possible to induce chiral magnetism by applying large electric fields in the paraelectric phase. For $E \geq 1 \mathrm{kV} / \mathrm{mm}$, this chiral scattering can be followed well above $T_{\mathrm{MF}}$ till the lock-in transition, but it cannot be ruled out that it even persists to higher temperatures. Note that this small chiral signal sits on top of the strong magnetic Bragg peak associated with the longitudinal spin-density wave (SDW) of $\mathrm{TbMnO}_{3}$ that exists between $T_{N}$ and $T_{\mathrm{MF}}$.

There are two orthogonal possibilities to add a $c$ polarized moment to the longitudinal SDW order existing between $T_{N}$ and $T_{\mathrm{MF}}$ in $\mathrm{TbMnO}_{3}$ : either out of phase or in phase with respect to the SDW $b$ moments, as it is illustrated in Figs. 1(f) and 1(g), respectively [30,31]. Only the out-of-phase mode results in cycloid order inducing ferroelectric polarization. The transition from the SDW to the multiferroic phase can, thus, be associated to the softening of that $c$-polarized magnon in the SDW phase [30,31]. The $c$-polarized moment (M_c) (length of the complex Fourier component) can be separated by the polarization analysis, where it forms the only contribution to the $\sigma_{\bar{y} y} \cong M_{c}{ }^{2}$ channel, while the $b$ component $M_{b}$ 
contributes entirely to $\sigma_{\bar{z} z} \cong M_{b}{ }^{2}$. The large $c$ component develops at the cycloid transition at $T_{\mathrm{MF}}$. However, we find finite but weak $M_{c}{ }^{2}$ even above $T_{\mathrm{MF}}$; see $\sigma_{y \bar{y}}$ shown in Fig. 1(c). We can quantitatively compare $M_{c}$ to the chiral scattering, which in the ideal cycloid configuration (at low temperature, almost perfectly realized at the chosen $\mathbf{Q}$ ) corresponds to $2 M_{b} M_{c}$. By poling in large electric fields, we obtain a nearly mono-domain state so that $\sigma_{\text {chir }}$ is obtained by the difference in the $\sigma_{\bar{x} x}$ and $\sigma_{x \bar{x}}$ channels. Figure 1(c) illustrates that below $T_{\mathrm{MF}}$ the chiral scattering $\left(\sigma_{x \bar{x}}-\sigma_{\bar{x} x}\right) / 2$ indeed corresponds to $2 M_{b} M_{c}=2 \sqrt{\sigma_{\bar{y} y} \sigma_{\bar{z} z}}$; the minor difference arises from the extinction reducing more strongly the observed $\sigma_{x \bar{x}}$. This relation is also fulfilled for the scattering persisting above $T_{\mathrm{MF}}$. From the two $c$-polarized modes shown in Figs. 1(f) and 1(g), only the out-of-phase mode condenses at the multiferroic transition inducing the chiral signal. If both modes condense in a similar way, $2 \sqrt{\sigma_{\bar{y} y} \sigma_{\bar{z} z}}$ must exceed the chiral scattering by a factor of $\sqrt{2}$. The chiral scattering observed above $T_{\mathrm{MF}}$, thus, corresponds to the soft and overdamped electromagnon mode depicted in Fig. 1(f), while the other $c$-polarized mode shown in Fig. 1(g) does not soften into diffuse scattering.

We also performed scans across the magnetic Bragg peak in two directions for temperatures above $T_{\mathrm{MF}}$, which are shown in Fig. S1 of the Supplemental Material [22]. The finite chiral component can be followed by subtracting the two channels corroborating the results obtained at the central Bragg positions. By comparing the Gaussian profiles fitted to the differences with those fitted to the total magnetic signal, we find that the chiral regions possess large coherence lengths, but the increase of the difference widths with the temperature indicates that the chiral correlation lengths are limited. The detailed temperature dependences and the full scans leave no doubt that $\mathrm{TbMnO}_{3}$ permits us to control chiral magnetism over a large temperature interval of about $10 \%$ above $T_{\mathrm{MF}}$.

We also studied the possibility to vary the chiral scattering by driving the electric field at constant temperature in $\mathrm{TbMnO}_{3}$. These hysteresis curves are shown in Fig. 2. Slightly above $T_{\mathrm{MF}}$, the hysteresis still exhibits a finite remanence, which is rapidly suppressed with increasing temperature. These measurements need considerable beam time due to the small chiral signals, and the remanent signals correspond to large time scales in consequence. The remanent signals seem not to be intrinsic to the multiferroic coupling but can arise from the anomalous $c$-axis relaxation reported for $\mathrm{TbMnO}_{3}$ [33]. At $32 \mathrm{~K}$, no sizeable chiral signal can be induced (with an electric field $E=1 \mathrm{kV} / \mathrm{mm}$ ).

Studies of chiral scattering above $T_{\mathrm{MF}}$ turned out more difficult in $\mathrm{MnWO}_{4}$ because the temperature extension of the controllable chirality is considerably smaller even when taking account of the overall smaller transition temperatures. Figure 3 shows that diffuse chiral scattering can be observed only for temperatures less than $0.1 \mathrm{~K}$ above $T_{\mathrm{MF}}$

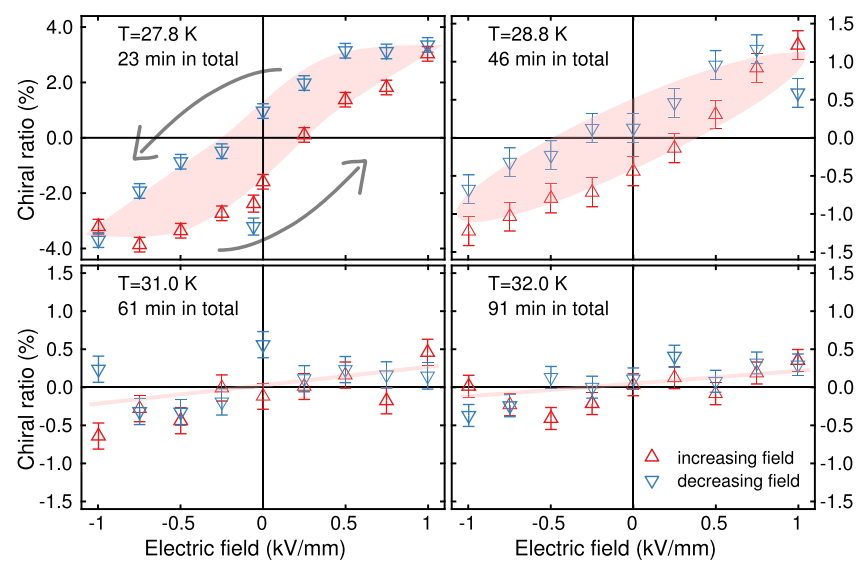

FIG. 2. Electric-field-driven hysteresis cycles of the chiral signal in $\mathbf{Q}=(2,0.28,1)$ above $T_{\mathrm{MF}}$ in $\mathrm{TbMnO}_{3}$. Weak chiral components can be controlled through the field with finite remanent signals.

with an applied electric field of $550(\mathrm{~V} / \mathrm{mm})$. There is finite chiral scattering above $T_{\mathrm{MF}}$ when rotating the sample away from the magnetic Bragg position [see Fig. 3(b)], indicating that the chiral response in $\mathrm{MnWO}_{4}$ at least partially arises from short-range correlations.

Applying a strong electric field to a ferroelectric material will increase the transition temperature. With the Ehrenfest relation [34] $\left(d T_{\mathrm{MF}} / d E\right)=\left[\left(\partial P_{\mathrm{FE}} / \partial T\right) /\left(\Delta C_{p} / T\right)\right]$, one can estimate the field-induced enhancement of the ferroelectric transition temperature in the two materials assuming a second-order improper ferroelectric phase transition. For $\mathrm{TbMnO}_{3}$, we obtain with $\left(\partial P_{\mathrm{FE}} / \partial T\right) \simeq 171\left(\mu \mathrm{C} / \mathrm{K} \mathrm{m}^{2}\right)[4]$ and $\left(\Delta C_{p} / T\right) \simeq 0.1\left(\mathrm{~J} / \mathrm{mol} \mathrm{K}^{2}\right) \simeq 2917\left(\mathrm{~J} / \mathrm{m}^{3} \mathrm{~K}^{2}\right) \quad$ [35] an enhancement of the ferroelectric transition by $\left(d T_{\mathrm{MF}} / d E\right)=0.06(\mathrm{Kmm} / \mathrm{kV})$, which is far too small to explain the observations of chiral scattering up to $\simeq 4 \mathrm{~K}$ above $T_{\mathrm{MF}}$. For $\mathrm{MnWO}_{4}$, the same analysis yields an even smaller electric-field-induced increase of $T_{\mathrm{MF}}$ by $\left(d T_{\mathrm{MF}} / d E\right)=0.003(\mathrm{~K} \mathrm{~mm} / \mathrm{kV})\left(\right.$ here $\left(\partial P_{\mathrm{FE}} / \partial T\right) \simeq$ $34\left(\mu \mathrm{C} / \mathrm{K} \mathrm{m}^{2}\right)$ and $\left.\left(\Delta C_{p} / T\right) \simeq 0.46\left(\mathrm{~J} / \mathrm{mol} \mathrm{K}^{2}\right)[20]\right)$. The

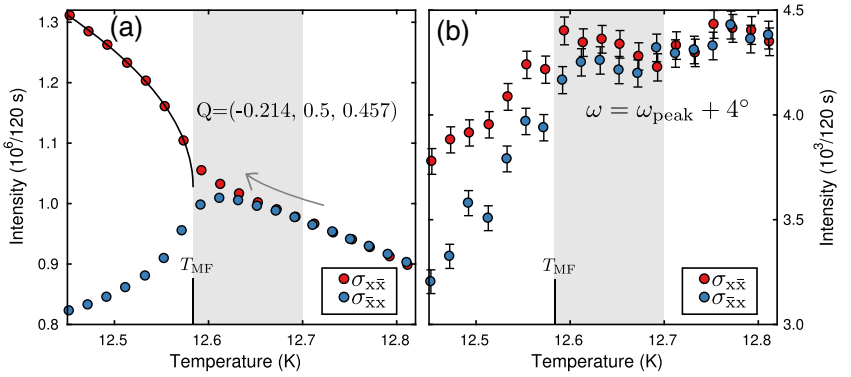

FIG. 3. Chiral magnetic scattering measured in $\mathrm{MnWO}_{4}$. Temperature dependence of $\sigma_{\bar{x} x}$ and $\sigma_{x \bar{x}}$ at the magnetic peak position $\mathbf{Q}=(-0.214,0.5,0.457)$ (a) and with the crystal rotated slightly away (by $4^{\circ}$ ) (b). The multiferroic transition temperature $T_{\mathrm{MF}}$ was determined by fitting a power law [32] to the count rate. An electric field of $550(\mathrm{~V} / \mathrm{mm})$ was applied to the sample along $b$ upon cooling. 
small enhancement of $T_{\mathrm{MF}}$ is just the consequence of the weak ferroelectric polarization $\boldsymbol{P}_{\mathrm{FE}}$, which results for $\mathrm{TbMnO}_{3}\left[\mathrm{MnWO}_{4}\right]$ in small energies per $\mathrm{Mn}$ site in an applied electric field $\boldsymbol{P}_{\mathrm{FE}} \boldsymbol{E} \frac{V_{\mathrm{uc}}}{n}=2 \times 10^{-4}\left(2 \times 10^{-5}\right) \mathrm{meV}$ at $E=1(\mathrm{kV} / \mathrm{mm})$, with $V_{\mathrm{UC}}$ the unit-cell volume and $n$ the number of formula units therein. These electric-fieldinduced energies are negligible compared to the thermal energies in both materials, $k_{B} T_{\mathrm{MF}}=2.4 \mathrm{meV}(1.1 \mathrm{meV})$. We may, thus, exclude that the persistence of chiral scattering above $T_{\mathrm{MF}}$ just arises from a field-induced shift of the transition temperature.

The precise analysis of the electric-field effect, however, has to take the splitting of the magnetic transitions into account involving two magnetic order parameters. Tolédano [36] and Tolédano et al. [32] have explicitly studied the symmetry conditions and developed the full Landau theory for the two materials $\mathrm{TbMnO}_{3}$ and $\mathrm{MnWO}_{4}$ studied here. The transition into the multiferroic phase combines properties of proper and improper ferroelectrics and is labeled as a pseudoproper ferroelectric transition [36]. Although the ferroelectric polarization describes the same symmetry reduction as the magnetic order parameter at $T_{\mathrm{MF}}$, it remains a secondary quantity because the transition from the SDW into the cycloid phase is driven by the magnetic interaction parameters. Because of the magnetoelectric coupling between ferroelectric polarization and the two magnetic order parameters, applying an electric field in the SDW phase will yield finite polarization that induces a finite value of the second magnetic order parameter and, thus, some long-range and static chiral components. However, as can already be seen in the sharp drop of the dielectric constant $[18,20,35]$, this effect is small and limited to temperatures very close to $T_{\mathrm{MF}}$, and it cannot explain the observed chiral components persisting above $T_{\mathrm{MF}}$ shown in Figs. 1 and 3. The quantitative comparison of this induced static and long-range chirality with our observation is discussed in detail in the Supplemental Material [22]. The chiral correlation we observe above $T_{\mathrm{MF}}$ cannot be static and long range, as in this case, the dielectric permittivity must be larger.

When poling the multiferroic domains with an external field, the entire domain volume enters the energy balance causing large effects. The same holds for the poling of short-range correlations above the long-range chiral and ferroelectric order, i.e., the experiments we perform. The energy gain for poling a cluster amounts to $\boldsymbol{P}_{\mathrm{FE}} \boldsymbol{E} \frac{4 \pi}{3} \xi_{\text {chir }}^{3}$ with the chiral correlation length $\xi_{\text {chir. }}$. This energy has to exceed the thermal energy in order to induce a significant imbalance of chiral fluctuations, which we sense through the polarized neutron diffraction. In first approximation, we may assume the local polarization in the fluctuation to be of similar size as that in the static multiferroic phase. For $\mathrm{MnWO}_{4}, \quad E \simeq 550(\mathrm{~V} / \mathrm{mm})$, and $P_{\mathrm{FE}}=60\left(\mu \mathrm{C} / \mathrm{m}^{2}\right)$ [18-20], and we, thus, obtain that a correlation length of at least $\simeq 100 \AA$ is needed to induce significant chiral magnetism slightly above $T=12.6 \mathrm{~K}$. Unfortunately, the chiral diffuse scattering is superposed to the much stronger Bragg scattering of the first magnetic transition in both systems so that it cannot be obtained from unpolarized experiments. For $\mathrm{MnWO}_{4}$, one may identify it with the diffuse scattering containing a $b$ component, whose correlation length can be extrapolated from the paramagnetic phase. It indeed exceeds $\simeq 100 \AA$ only in a tiny temperature range around $T_{\mathrm{MF}}$ [37]. Dielectric spectroscopy observes a sharp softening of the relaxation rate close to the ferroelectric transition in $\mathrm{MnWO}_{4}$ [38]. The energy resolution of our $\mathrm{MnWO}_{4}$ neutron experiment on IN14 corresponds to $\simeq 30 \mathrm{GHz}$ half width at half maximum so that any slower relaxation is seen as an elastic signal. Only sufficiently close to $T_{\mathrm{MF}}$, the fluctuations appear in the time window of our quasistatic experiment. Extrapolating the dielectric spectroscopy results [38] to $30 \mathrm{GHz}$ yields $\Delta T=0.6 \mathrm{~K}$ larger than the temperature interval, where chiral scattering can be poled in $\mathrm{MnWO}_{4}$ (see Fig. 3), as in addition the correlation length of the fluctuation needs to be sufficiently large. We may conclude that the ferroelectric cycloid transition in $\mathrm{MnWO}_{4}$ corresponds to the soft, overdamped electromagnon, which possesses both an electric and a chiral magnetic component [31]. Sufficiently close to $T_{\mathrm{MF}}$, this electromagnon is observed as a quasistatic fluctuation that can be controlled by the external electric field.

The quantitative analysis of the chiral fluctuations in $\mathrm{TbMnO}_{3}$ is more difficult. Dielectric spectroscopy observes a strong $c$-axis relaxation already at temperatures much above $T_{N}$, whose origin remains unclear [33]. This relaxation will impact any electric-field experiments in $\mathrm{TbMnO}_{3}$. In particular, the control of multiferroic polar components will follow or be pinned by these relaxation processes. $\mathrm{TbMnO}_{3}$ exhibits a ferroelectric polarization of $P_{\mathrm{FE}}=600\left(\mu \mathrm{C} / \mathrm{m}^{2}\right)$ [4], about 1 order of magnitude larger than that in $\mathrm{MnWO}_{4}$. However, the correlation length enters the energy balance in the third power so that the lower limit for stabilizing fluctuations around the higher $T_{\mathrm{MF}}$ is comparable to that in $\mathrm{MnWO}_{4}, \simeq 100 \AA$. The scans shown in Fig. S1 of the Supplemental Material [22] indicate that slightly above $T_{\mathrm{MF}}$, correlation lengths exceed this value. In the picture of a softening electromagnon fluctuation driving the ferroelectric transition, the fluctuations must posses such large correlation lengths as well as a low relaxation rate over the broad observed temperature range.

It has been proposed that chiral type-II multiferroics exhibit a distinct phase between the Néel temperature and the onset of long-range chiral magnetic and ferroelectric order [39]. This phase is characterized by fixed chirality without long-range order, and it is called the cholesteric phase. This symmetry reduction bears similarities with the Ising nematic phase discussed, for example, in Fe-based superconductors [40]. For $\mathrm{TbMnO}_{3}$, it appears tempting to identify the disappearance of the poled chiral scattering with the lock-in transition, but it cannot be ruled out that 
chiral poling even persists above $T_{\text {lock-in }}$ and that the considerable difference between the two studied multiferroics is coupled to the strong relaxation phenomena observed only in $\mathrm{TbMnO}_{3}$ [33].

In conclusion, we have studied chiral diffuse scattering at temperatures above the zero-field transition to the longrange chiral and multiferroic order. For $\mathrm{MnWO}_{4}$, we can connect the transition into the multiferroic state with the softening and overdamping of an electromagnon fluctuation that exhibits the necessary long correlation lengths and low energies only in a tiny temperature interval close to $T_{\mathrm{MF}}$. In $\mathrm{TbMnO}_{3}$, one can pole and control chiral magnetism in a much larger temperature interval, possibly related to the strong $c$-axis relaxation observed in dielectric spectroscopy.

In both multiferroic materials, it is, thus, possible to pole and control short-range chiral magnetism by a moderate electric field above the long-range multiferroic phase.

This study was supported by the Deutsche Forschungsgemeinschaft through CRC 1238 projects A02 and B04. We thank J. Hemberger, T. Lorenz, and C. Papas for stimulating discussions and E. Bourgeat-Lami, E. Lelièvre-Berna, and Y. Memphis for developing a special sample can, which enormously facilitated our experiments.

*braden@ph2.uni-koeln.de

${ }^{\dagger}$ Present address: Fraunhofer INT, Euskirchen, Germany.

[1] T. Kimura, S. Ishihara, H. Shintani, T. Arima, K. Takahashi, K. Ishizaka, and Y. Tokura, Nature (London) 426, 55 (2003).

[2] S. W. Cheong and M. Mostovoy, Nat. Mater. 6, 13 (2007); D. Khomskii, Physics 2, 20 (2009); K. F. Wang, J.-M. Liu, and Z. F. Ren, Adv. Phys. 58, 321 (2009); S. Dong and J.-M. Liu, Mod. Phys. Lett. B 26, 1230004 (2012); Y. Tokura, S. Seki, and N. Nagaosa, Rep. Prog. Phys. 77, 076501 (2014).

[3] T. Goto, T. Kimura, G. Lawes, A. P. Ramirez, and Y. Tokura, Phys. Rev. Lett. 92, 257201 (2004).

[4] T. Kimura, G. Lawes, T. Goto, Y. Tokura, and A. P. Ramirez, Phys. Rev. B 71, 224425 (2005).

[5] N. Aliouane, K. Schmalzl, D. Senff, A. Maljuk, K. Prokes, M. Braden, and D. N. Argyriou, Phys. Rev. Lett. 102, 207205 (2009).

[6] Y. Yamasaki, H. Sagayama, T. Goto, M. Matsuura, K. Hirota, T. Arima, and Y. Tokura, Phys. Rev. Lett. 98, 147204 (2007).

[7] H. Sagayama, K. Taniguchi, N. Abe, T. H. Arima, M. Soda, M. Matsuura, and K. Hirota, Phys. Rev. B 77, 220407(R) (2008).

[8] S. Seki, Y. Yamasaki, M. Soda, M. Matsuura, K. Hirota, and Y. Tokura, Phys. Rev. Lett. 100, 127201 (2008).

[9] T. Finger, D. Senff, K. Schmalzl, W. Schmidt, L. P. Regnault, P. Becker, L. Bohatý, and M. Braden, Phys. Rev. B 81, 054430 (2010).
[10] T. Finger, D. Senff, K. Schmalzl, W. Schmidt, L. P. Regnault, P. Becker, L. Bohatý, and M. Braden, J. Phys. Conf. Ser. 211, 012001 (2010).

[11] I. Cabrera, M. Kenzelmann, G. Lawes, Y. Chen, W. C. Chen, R. Erwin, T. R. Gentile, J. B. Leão, J. W. Lynn, N. Rogado, R. J. Cava, and C. Broholm, Phys. Rev. Lett. 103, 087201 (2009).

[12] J. Stein, M. Baum, S. Holbein, T. Cronert, V. Hutanu, A. C. Komarek, and M. Braden, J. Phys. Condens. Matter 27, 446001 (2015).

[13] F. Fabrizi, H. C. Walker, L. Paolasini, F. de Bergevin, A. T. Boothroyd, D. Prabhakaran, and D. F. McMorrow, Phys. Rev. Lett. 102, 237205 (2009).

[14] E. Schierle, V. Soltwisch, D. Schmitz, R. Feyerherm, A. Maljuk, F. Yokaichiya, D. N. Argyriou, and E. Weschke, Phys. Rev. Lett. 105, 167207 (2010).

[15] T. Kubacka et al., Science 343, 1333 (2014).

[16] A. Glavic, C. Becher, J. Voigt, E. Schierle, E. Weschke, M. Fiebig, and T. Brückel, Phys. Rev. B 88, 054401 (2013).

[17] Y. Yamaguchi, T. Nakano, Y. Nozue, and T. Kimura, Phys. Rev. Lett. 108, 057203 (2012).

[18] K. Taniguchi, N. Abe, T. Takenobu, Y. Iwasa, and T. Arima, Phys. Rev. Lett. 97, 097203 (2006).

[19] O. Heyer, N. Hollmann, I. Klassen, S. Jodlauk, L. Bohatý, P. Becker, J. Mydosh, T. Lorenz, and D. Khomskii, J. Phys. Condens. Matter 18, L471 (2006).

[20] A. H. Arkenbout, T. T. M. Palstra, T. Siegrist, and T. Kimura, Phys. Rev. B 74, 184431 (2006).

[21] M. Baum, J. Leist, Th. Finger, K. Schmalzl, A. Hiess, L. P. Regnault, P. Becker, L. Bohatý, G. Eckold, and M. Braden, Phys. Rev. B 89, 144406 (2014).

[22] See Supplemental Material at http://link.aps.org/ supplemental/10.1103/PhysRevLett.119.177201 for the description of experimental details and additional data, which includes Refs. [23,24]

[23] K. Schmalzl, W. Schmidt, S. Raymond, H. Feilbach, C. Mounier, B. Vettard, and T. Brückel, Nucl. Instrum. Methods Phys. Res., Sect. A 819, 89 (2016).

[24] https://www.ill.eu/fr/instruments-support/computing-forscience/cs-software/all-software/matlab-ill/rescal-formatlab/.

[25] P. J. Brown, in Neutron Scattering from Magnetic Materials, edited by T. Chatterji (Elsevier B. V., Amsterdam, 2006).

[26] D. Meier, N. Aliouane, D. N. Argyriou, J. A. Mydosh, and T. Lorenz, New J. Phys. 9, 100 (2007).

[27] M. Kenzelmann, A. B. Harris, S. Jonas, C. Broholm, J. Schefer, S. B. Kim, C. L. Zhang, S.-W. Cheong, O. P. Vajk, and J. W. Lynn, Phys. Rev. Lett. 95, 087206 (2005).

[28] R. Kajimoto, H. Yoshizawa, H. Shintani, T. Kimura, and Y. Tokura, Phys. Rev. B 70, 012401 (2004).

[29] S. B. Wilkins, T. R. Forrest, T. A. W. Beale, S. R. Bland, H. C. Walker, D. Mannix, F. Yakhou, D. Prabhakaran, A. T. Boothroyd, J. P. Hill, P. D. Hatton, and D. F. McMorrow, Phys. Rev. Lett. 103, 207602 (2009).

[30] D. Senff, P. Link, K. Hradil, A. Hiess, L. P. Regnault, Y. Sidis, N. Aliouane, D. N. Argyriou, and M. Braden, Phys. Rev. Lett. 98, 137206 (2007).

[31] D. Senff, N. Aliouane, D. N. Argyriou, A. Hiess, L. P. Regnault, P. Link, K. Hradil, Y. Sidis, and M. Braden, J. Phys. Condens. Matter 20, 434212 (2008). 
[32] P. Tolédano, B. Mettout, W. Schranz, and G. Krexner, J. Phys. Condens. Matter 22, 065901 (2010).

[33] F. Schrettle, P. Lunkenheimer, J. Hemberger, V. Yu. Ivanov, A. A. Mukhin, A. M. Balbashov, and A. Loidl, Phys. Rev. Lett. 102, 207208 (2009).

[34] Y. Ishibashi and I. Suzuki, J. Phys. Soc. Jpn. 53, 3466 (1984).

[35] D. O’Flynn, M. R. Leed, and G. Balakrishnan, J. Phys. Condens. Matter 26, 256002 (2014).
[36] P. Tolédano, Phys. Rev. B 79, 094416 (2009).

[37] S. Holbein, Ph.D. dissertation, Universität zu Köln, 2016.

[38] D. Niermann, C.P. Grams, P. Becker, L. Bohatý, H. Schenck, and J. Hemberger, Phys. Rev. Lett. 114, 037204 (2015).

[39] S. Onoda and N. Nagaosa, Phys. Rev. Lett. 99, 027206 (2007).

[40] R. M. Fernandes, A. V. Chubukov, and J. Schmalian, Nat. Phys. 10, 97 (2014). 